\section{Dispersible silica nanoparticles as carrier for enhanced bioactivity of chlorfenapyr}

\author{
Mei-Rong Song,${ }^{\dagger}$ Shu-Min CuI, ${ }^{\dagger}$ Fei GaO, ${ }^{\dagger}$ \\ Yu-Ru Liu, ${ }^{\dagger}$ Cai-Ling Fan, ${ }^{*}{ }^{\dagger}$ \\ Tian-Qian LeI ${ }^{\dagger \dagger}$ and De-Chang LIU ${ }^{\dagger \dagger}$
}

${ }^{\dagger}$ Henan Agricultural University, Zhengzhou 450002, P. R. China ${ }^{\dagger \dagger}$ Kaifeng Bokai Bicaro Biochemical Co., Ltd., Kaifeng 475000, P. R. China

(Received April 10, 2012; Accepted May 14, 2012)

Keywords: silica nanoparticles, nanopesticides, chlorfenapyr.

Electronic supplementary materials The online version of this article contains supplementary materials (Supplemental Figure S1, Supplemental Tables S1 and S2), which is available at http://www.jstage.jst.go.jp/browse/ jpestics/.

Pesticides are being used in agriculture to control pests for better yield and production. However, due to evaporation, rain waste-out, photodegradation, hydrolysis and other factors, most pesticides are lost before they reach their targets. ${ }^{1)}$ Extensive quantities of pesticides are used to compensate for the losses that lead to serious environmental pollution and human toxicity. ${ }^{1,2)}$ An effective way to solve these problems is nanotechnology. The increase in the surface to volume ratio and surface energy of nanopesticides facilitates the penetration and attachment of an effective agent on the surface of plant. As a result, the efficacy could be significantly increased by the application of nanopesticides. ${ }^{3,4)}$ In some cases, controlled release technology can integrate into the advantages of nanopesticides. ${ }^{5)}$ Therefore, it is believed that the production of nanopesticides will result in the use of smaller amount and fewer applications. ${ }^{3)}$ Nanopesticides are environmentally friendly and provide new opportunities and wide application prospects for pesticide research. ${ }^{3)}$

Recently, the use of silica nanoparticles in pesticides has been attracting progressively more attention. ${ }^{6,7)}$ They have been shown to be biocompatible and has potential as drug delivery vehicles for medical and veterinary treatments. Hollow and mesoporous silica nanoparticles have also been developed as carriers. ${ }^{8-10)}$ Experiments have demonstrated that they are effective

\footnotetext{
* To whom correspondence should be addressed.

E-mail:smr770505@yahoo.com.cn

Published online July 11, 2012

(c) Pesticide Science Society of Japan
}

in the protection against UV degradation and controlled release dependently on the pore diameter and shell thickness. ${ }^{8,9)}$ However, the cost and industrialization of the carrier is usually the main impediment to the application of a nanopesticide. Therefore, to accelerate the application of nanopesticides, inexpensive and commercialized carriers are required, and related studies are important.

In research, ${ }^{\text {s) }}$ both hydrophilic and hydrophobic silica nanoparticles have been reported to provide insecticide activity on their own. Dispersible silica nanoparticles have been inexpensively commercialized for years. These nanoparticles were modified with an organic surfactant that made them dispersible into organic solvent, ${ }^{11)}$ enhancing the ability of a carrier to load a hydrophobic drug because the enormous surface-to-volume ratio will not be wasted by aggregation. In this paper, an effective chlorfenapyr nanoformulation was prepared based on the above carrier.

In a typical drug-loading process, $0.5 \mathrm{~g}$ of silica nanoparticles and $2.0 \mathrm{~g}$ of chlorfenapyr are added to acetone. The mixture is then stirred continuously by a magnetic follower at room temperature. A white turbid suspension appears, and the process continues for $30 \mathrm{~min}$ to ensure maximum drug loading. The white turbid suspension is centrifuged and washed with ethanol. The final product is a white powder that has been dried in air at $40^{\circ} \mathrm{C}$ for $24 \mathrm{hr}$ and ground in a ceramic pestle and mortar.

The morphology and size of chlorfenapyr-loaded silica nanoparticles were measured with a JEM 2100 transmission electron microscopy (TEM) instrument. As shown in Fig. 1, the size of chlorfenapyr-loaded silica particles is in the range of 50-200 nm. A magnified picture shows that they consist of several silica nanoparticles that congregate (inset in Fig. 1), and the surrounding entanglement should be ascribed to the drug chlorfenapyr. TEM demonstrated that the resulting drug-loaded particles are nanosized and dispersible without serious aggregation.

The Fourier transform infrared (FTIR) of chlorfenapyr-loaded silica particles was recorded with a CARY Eclipse FTIR spectrophotometer and compared with those of pure chlorfenapyr and the carrier silica nanoparticles alone. Nearly all characteristic peaks of chlorfenapyr-loaded silica particles were found to be identical to those of the pure drug except for the broad absorption peak between $1400-1000 \mathrm{~cm}^{-1}$, which might be the integrated adsorption of silica and chlorfenapyr. This result indicates that the drug might be physically adsorbed on the carrier.

The actual drug-loading weight was determined with an American Diamond thermal gravimetric analyzer (TGA). The samples were heated at a rate of $10^{\circ} \mathrm{C} / \mathrm{min}$ in a stream of nitrogen gas. The results are shown in Fig. 2. The calculation process of the actual drug loading weight is according to the following equation described by $\mathrm{Lu}^{12}$ 


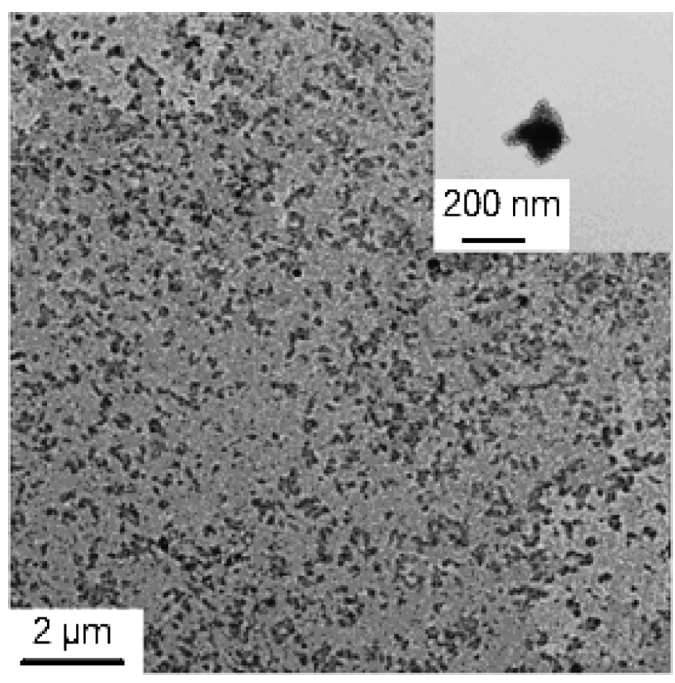

Fig. 1. TEM image of chlorfenapyr-loaded silica nanoparticles.

$$
\frac{B-W_{1}}{1 \text { or } 100 \%-B}=\frac{T-W-W_{2}}{1 \text { or } 100 \%-T}
$$

$W_{1}$ and $W_{2}$ represent the percentage weight losses of physically and chemically adsorbed water $\left(<150^{\circ} \mathrm{C}\right)$ in blank silica nanoparticles and chlorfenapyr-loaded silica particles, respectively; $B$ is the percentage weight loss corresponding to the adsorption of water and organic content of silica nanoparticles, and $T$ represents the percentage total weight loss of adsorbed water and the organic part of chlorfenapyr-loaded silica particles. By substituting the equation with the following: $B=8.92 \%$, $W_{1}=4.40 \%, T=44.29 \%, W_{2}=1.75 \%$, the value of $\mathrm{W}$ is calculated to be $39.78 \%$.

In addition, the chlorfenapyr-loaded silica nanoparticles are not the mixture of chlorfenapyr and silica nanoparticles since the beginning decomposing temperature of the chlorfenapyrloaded silica particles is $200^{\circ} \mathrm{C}$, higher than that of the pure drug. This indicates that there is an interaction between the drug and the carrier that is ascribed to the hydrophobic force.

The suspension concentrate of nanosized chlorfenapyr (NCSC) was prepared by high-speed shearing a mixture of chlorfenapyr-loaded nanoparticles, dispersant, antifoam, thickener, preservative, antifreezer and water. The suspension concentrate of microsized chlorfenapyr (MCSC) (CHU-JIN) with an average size of $2.5-3.5 \mu \mathrm{m}$ and $10-$ wt.\% effective content supplied by the BASF AG Company was examined.

The insecticidal toxicity tests of NCSC and MCSC were compared using bollworm larvae $(\sim 3 \mathrm{mg} /$ larva $)$ as a model insect and carried out by the diet incorporation method. ${ }^{13,14)}$ Bollworm larvae were reared on an artificial $\operatorname{diet}^{15)}$ at $27 \pm 1^{\circ} \mathrm{C}$ with a

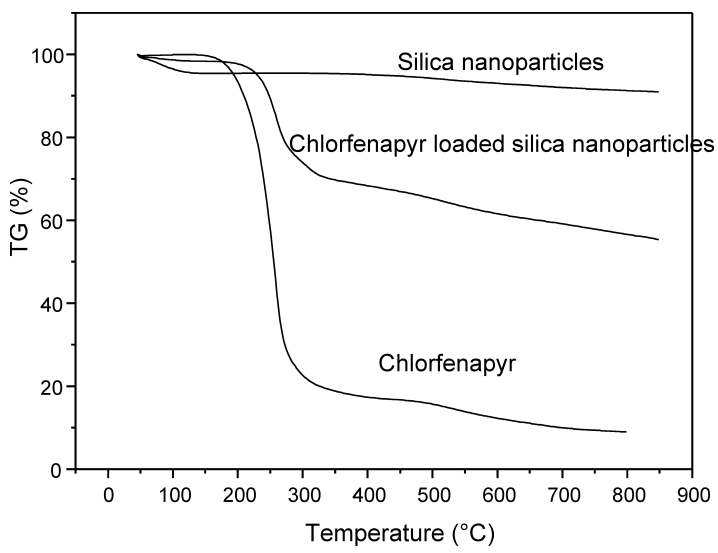

Fig. 2. TGA of dispersible silica nanoparticles, chlorfenapyr, and chlorfenapyr-loaded silica nanoparticles.

photoperiod of 14 -hr light and 10 -hr dark, and $60-80 \%$ relative humidity (RH). The mixed medium of the artificial diet and the two SCs were prepared with chlorfenapyr at different concentrations of $1.68,3.38,6.75,12.5,25,50$ and $100 \mu \mathrm{g} / \mathrm{mL}$. The control consisted of a normal meridic diet (without toxins). The mortality data were analyzed by a Statistical Product and Service Solutions (SPSS) program. The concentrations showing corrected mortality between $20 \%$ and $80 \%$ at $72 \mathrm{hr}$ were used for the calculation of the median lethal concentration $\left(\mathrm{LC}_{50}\right)$.

The three-day $\mathrm{LC}_{50}$ was estimated for each formulation and expressed in terms of the concentration $(\mu \mathrm{g} / \mathrm{mL})$. The results of the insecticidal toxicity for MCSC and NCSC on cotton bollworm in laboratory tests are shown in Table 1 . The $\mathrm{LC}_{50}$ of NCSC is 2.25 times that of MCSC, which indicated that the insecticidal activity was significantly enhanced by using silica nanoparticles as the carrier.

The control effect of MCSC and NCSC against plutella xylostellas was carried in (Brassica chinese) field. When the dose of NCSC was half of that of MCSC, the corrected controlling effect of NCSC was higher than that of MCSC after 1 day and the same in the following two days. This result suggests that the dosage of NCSC can be decreased by $50 \%$ to achieve the same control effect of MCSC.

The enhancing biological efficacy of chlorfenapyr-loaded silica nanoformulation might first be related to the nanosize, and its advantages are reviewed at the beginning of the paper. Secondly, silica nanoparticles provide insecticide activity, as Goswami ${ }^{7)}$ reported. A possible combined action is now being investigated in our laboratory; however, it is not easy since chlorfenapyr-loaded silica nanoparticles with different loading weight over a wide range could not be prepared as an option.

Table 1. Toxicity for MCSC and NCSC on the cotton bollworm in a laboratory test

\begin{tabular}{clccc}
\hline Samples & Toxic regression equation & $r$ & LC $_{50}$ & Toxicity ratio \\
\hline MCSC & $y=0.8387+5.4413 x$ & 0.8966 & $5.82 \mu \mathrm{g} / \mathrm{mL}$ & 1 \\
NCSC & $y=3.7047+3.139 x$ & 0.9533 & $2.59 \mu \mathrm{g} / \mathrm{mL}$ & 2.25 \\
\hline
\end{tabular}


In conclusion, an effective chlorfenapyr nanoformulation by using dispersible silica nanoparticles as a carrier was prepared. Both laboratory and field tests demonstrated that the insecticidal toxicity of nanoformulation is twice as higher as that of the microformulation. Therefore, in this paper, we provide significant basic data for the future applications of nanopesticides.

\section{Acknowledgements}

This work was supported by a scientific and technological project of the Henan Province of China (122102210051).

\section{References}

1) M. Y. Arica, M. Yiğitoğlu and M. Lale, F. N. KöK and V. Hasirei: Turk. J. Chem. 21, 100-104 (1997).

2) A. Roy, J. Bajpai and A. K. Bajpai: Indian J. Chem. Technol. 16, 388395 (2009).

3) L. Jiang, F. Zheng, P. F. Leng, M. Xu and M. J. Zhao: Guangdong Agric. Sci. 97-100 (2010).

4) H. Zhang, D. Wang, R. Butler, N. L. Campbell, J. Long, B. Tan, D. J. Duncalf, A. J. Foster, A. Hopkinson, D. Taylor, D. Angus, A. I.
Cooper and S. P. Rannard: Nat. Nanotechnol. 3, 506-511 (2008).

5) M. Z. Hussein, Z. Zainal, A. H. Yahaya and D. W. V. Foo: J. Control. Release 82, 417-427 (2002)

6) A. G. S. Prado, A. O. Moura and A. R. Nunes: J. Agric. Food Chem. 59, 8847-8852 (2011).

7) A. Goswami, I. Roy, S. Sengupta and N. Debnath: Thin Solid Films 519, 1252-1257 (2010).

8) F. Liu, L.-X. Wen, Z.-Z. Li, W. Yu, H.-Y. Sun and J.-F. Chen: Mater. Res. Bull. 41, 2268-2275 (2006).

9) Z.-Z. Li, S.-A. Xu, L.-X. Wen, F. Liu, A. Q. Liu, Q. Wang, H. Y. Sun, W. Yu and J. F. Chen: J. Control. Release 111, 81-88 (2006).

10) F. Torney, B. G. Trewyn, V. S.-Y. Lin and K. Wang: Nat. Nanotechnol. 2, 295-300 (2007).

11) Z. J. Zhang, Z. Cao, X. H. Li and Z. L. Du: CN patent No. ZL 20041 0010304.2

12) C. X. (Cynthia) Lin, S. Z. Qiao, C. Z. Yu, S Ismadji and G. Q. (Max) Lu: Microporous Mesoporous Mater. 117, 213-219 (2009).

13) G. T. Gujar, A. Kumari, V. Kalia and K. Chandrashekar: Curr. Sci. 78, 995-1001 (2000).

14) G. T. Gujar, R. Nair, B. P. Singh, A. Kumari and V. Kalia: Crop Prot. 27, 537-544 (2008). 